\title{
A Novel Hap1-Tsc1 Interaction Regulates Neuronal mTORC1 Signaling and Morphogenesis in the Brain
}

\author{
Luis A. Mejia, ${ }^{1,2}$ Nadia Litterman, ${ }^{1,2 \star}$ Yoshiho Ikeuchi, ${ }^{1,3 *}$ Luis de la Torre-Ubieta, ${ }^{1,2}$ Eric J. Bennett, ${ }^{4}$ Chi Zhang, ${ }^{1}$ \\ J. Wade Harper, ${ }^{4}$ and Azad Bonni ${ }^{1,2,3}$ \\ ${ }^{1}$ Department of Neurobiology, Harvard Medical School, Boston, Massachusetts 02115, ${ }^{2}$ Program in Neuroscience, Harvard Medical School, Boston, \\ Massachusetts 02115 , ${ }^{3}$ Department of Anatomy and Neurobiology, Washington University School of Medicine, St. Louis, Missouri 63110, and ${ }^{4}$ Department \\ of Cell Biology, Harvard Medical School, Boston, Massachusetts 02115
}

Tuberous sclerosis complex (TSC) is a leading genetic cause of autism. The TSC proteins Tsc1 and Tsc2 control the mTORC1 signaling pathway in diverse cells, but how the mTORC1 pathway is specifically regulated in neurons remains to be elucidated. Here, using an interaction proteomics approach in neural cells including neurons, we uncover the brain-enriched protein huntingtin-associated protein 1 (Hap1) as a novel functional partner of Tsc1. Knockdown of Hap1 promotes specification of supernumerary axons in primary hippocampal neurons and profoundly impairs the positioning of pyramidal neurons in the mouse hippocampus in vivo. The Hap 1 knockdown-induced phenotypes in primary neurons and in vivo recapitulate the phenotypes induced by Tscl knockdown. We also find that Hap1 knockdown in hippocampal neurons induces the downregulation of Tsc1 and stimulates the activity of mTORC1, as reflected by phosphorylation of the ribosomal protein S6. Inhibition of mTORC1 activity suppresses the Hap1 knockdown-induced polarity phenotype in hippocampal neurons. Collectively, these findings define a novel link between Hap1 and Tsc1 that regulates neuronal mTORC1 signaling and neuronal morphogenesis, with implications for our understanding of developmental disorders of cognition.

\section{Introduction}

Tuberous sclerosis complex (TSC) is a developmental disorder and leading genetic cause of autism. Neurological manifestations of TSC include epilepsy, autistic disorder, intellectual disability (ID), learning disabilities, and behavioral disturbances (Orlova and Crino, 2010). Because our understanding of the pathogenesis of TSC is poor, treatment options for patients with these disorders remain inadequate.

Mutations of the TSC1 or TSC2 genes cause TSC (Orlova and Crino, 2010). The TSC1 and TSC2 proteins centrally regulate the mTORC1 signaling pathway. The TSC1-TSC2 dimer inhibits the mTORC1 activator, Rheb. Loss of TSC1-TSC2 function activates the mTORC1 pathway, which normally drives protein synthesis in response to diverse signals (Ma and Blenis, 2009). Deregulated protein synthesis stemming from perturbation of the mTORC1 pathway is associated with seizures and learning and behavior

Received May 27, 2013; revised 0ct. 1, 2013; accepted Oct. 7, 2013.

Author contributions: L.A.M., N.L., Y.I., L.d.I.T.-U., J.W.H., and A.B. designed research; L.A.M., N.L., Y.I., L.d.I.T.-U., E.J.B., and C.Z. performed research; L.A.M., N.L., Y.I., L.d.I.T.-U., and E.J.B. analyzed data; L.A.M. and A.B. wrote the paper.

This work was supported by National Institutes of Health Grant NS041021 to A.B., a Harvard-Roche grant to A.B., and F31 National Research Service Award fellowship NS068037 to L.A.M. We thank Mathew E. Sowa and John R. Lydeard for expert help with MS and A.B. laboratory members for helpful discussions.

The authors declare no competing financial interests.

*N.L. and Y.I. contributed equally to this work.

Correspondence should be addressed to Dr. Azad Bonni, Department of Anatomy and Neurobiology, Washington University School of Medicine, St. Louis, M0 63110. E-mail: bonni@wustl.edu.

E.J. Bennett's current address: Division of Biological Sciences, University of California, San Diego, La Jolla, CA 92093.

DOI:10.1523/JNEUROSCI.2290-13.2013

Copyright $\odot 2013$ the authors $\quad 0270-6474 / 13 / 3318015-07 \$ 15.00 / 0$ deficits in animals, and mutations in TSC1, TSC2, PTEN, eIF4E, and FMRP in autism and ID suggest that deregulation of the mTORC1 pathway plays a role in the pathogenesis of these brain disorders (Santini et al., 2013). However, how the mTORC1 pathway is specifically regulated in neurons remains largely unexplored.

The advent of computation-assisted interaction proteomics led us to systematically search for binding partners of autism spectrum disorder (ASD)- and ID-linked proteins. Using multiple parallel affinity purifications and the CompPASS analysis platform (Sowa et al., 2009), we applied an immunoprecipitation-mass spectrometry (IP-MS) approach in neurons. Here, we identify the protein Hap1 as a novel binding partner of Tsc1. Hap1, a $67 \mathrm{kDa}$ or $70 \mathrm{kDa}$ protein (A or B rodent isoforms, respectively), was originally identified as an interactor of the protein Huntingtin (Li et al., 1995). Hap1 may regulate the trafficking of microtubule- and membrane-associated protein cargoes in neurons. In this function, Hapl is thought to promote protein recycling and prevent protein degradation (Rong et al., 2007; Sheng et al., 2008). Interestingly, Hap1 expression appears to be largely limited to neural tissues (Dragatsis et al., 2000). We found that Hap1 knockdown promotes the specification of supernumerary axons in hippocampal neurons, and Hap1 knockdown in the developing hippocampus impairs the positioning of pyramidal neurons in vivo, mimicking the effect of Tsc1 knockdown. In biochemical assays, Hap1 knockdown triggers the downregulation of endogenous neuronal Tsc1 and stimulates phosphorylation of the mTORC1-regulated ribosomal protein S6. In epistasis analyses, inhibition of mTORC1 by rapamycin or an active form of Tsc2 suppresses the Hap1 knockdown-induced phenotype in neurons. Together, these data identify a novel function for Hap 1 in the regu- 


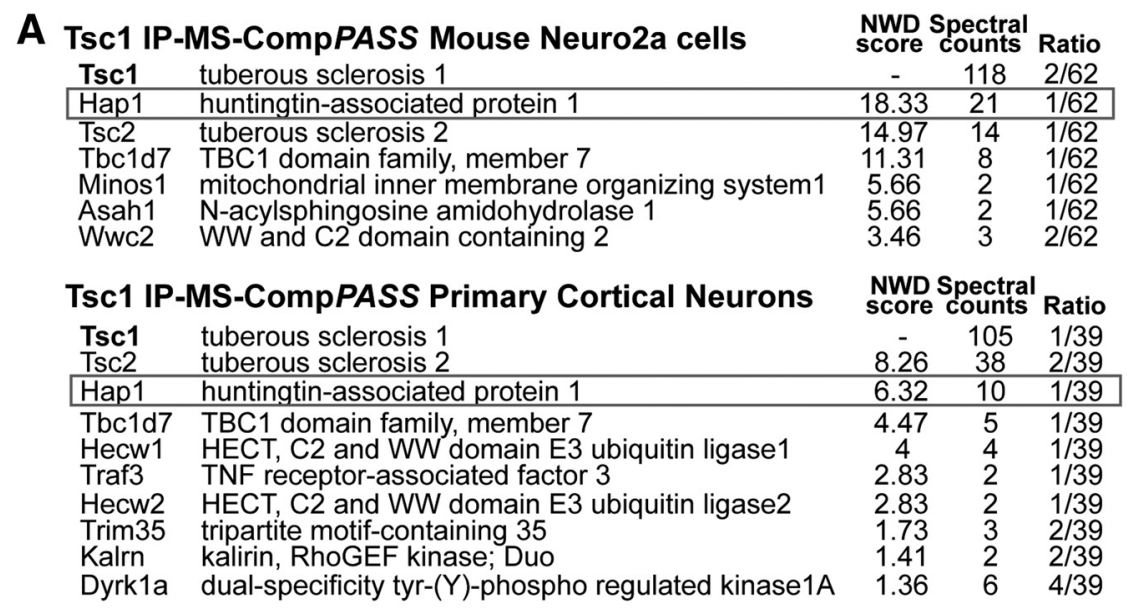

\section{C}

E18 mouse brain

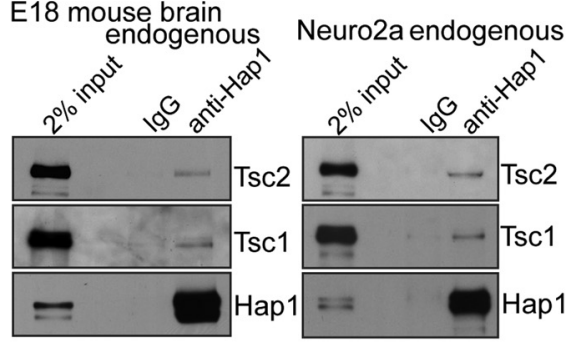

B
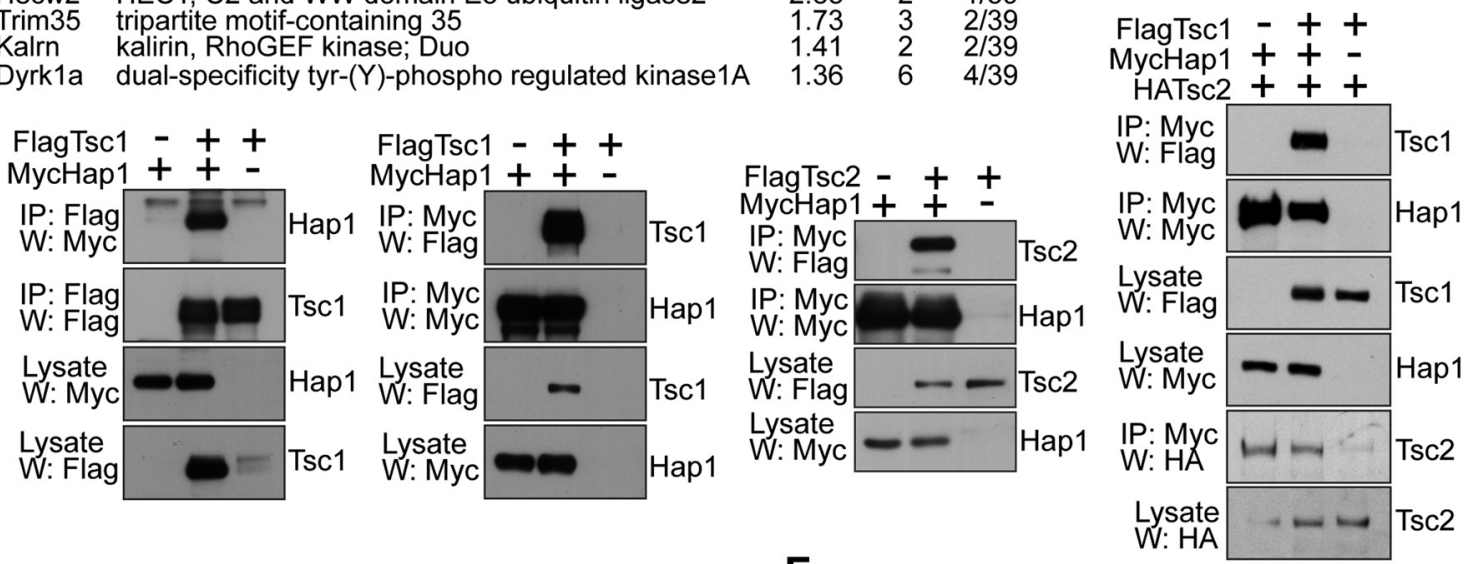

D

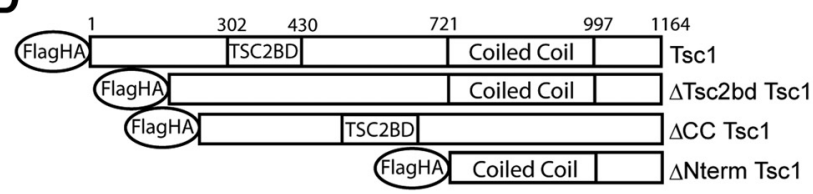
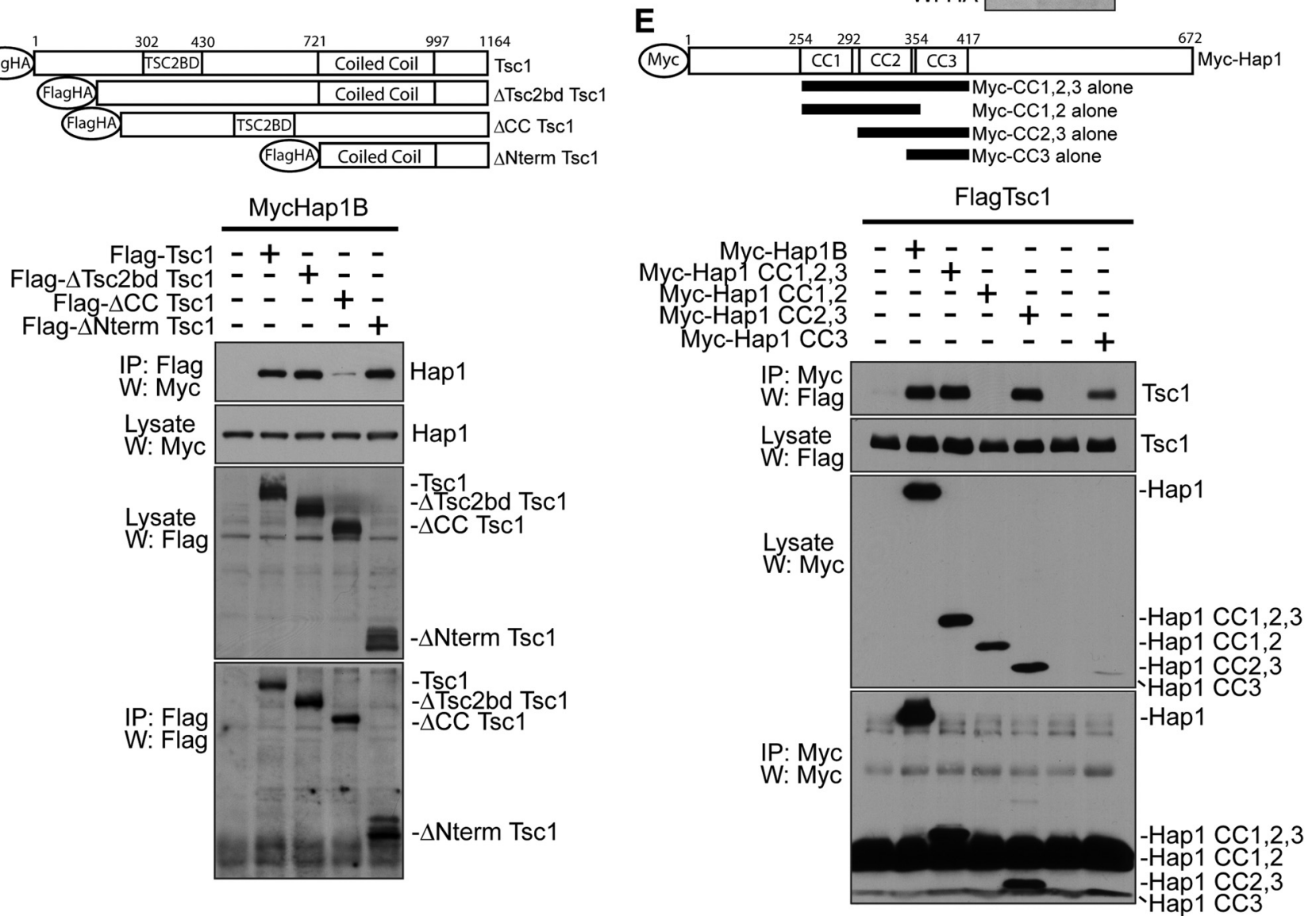

Figure 1. Identification of Hap1 as a novel Tsc1-interacting protein. A, IP-MS-CompPASS of Tsc1 in neuro2a and cortical neurons. Lysates of stable FLAG-Tsc1-expressing neuro2a or FLAG-Tsc1 lentivirus-infected cortical neurons were FLAG resin-immunoprecipitated. Bait complexes were analyzed by LC MS/MS. Analysis was done in CompPASS against dedicated neuro2a or cortical neuron IP-MS databases. High-confidence interacting proteins had normalized weighted D (NWD) scores $>1$. Total appearances across unrelated parallel IP-MS bait-runs are shown ("Ratio"). "Spectral counts" represent the number of tryptic peptides detected by the mass spectrometer corresponding to each protein. B, Reciprocal coimmunoprecipitations of Hap1 and (Figure legend continues.) 
lation of neuronal mTORC1 signaling and consequent neuronal morphogenesis.

\section{Materials and Methods}

All animal work was approved by the Harvard Institutional Animal Care and Use Committee.

Immunoprecipitation-mass spectrometry (IP-LC-MS/MS). IP-MS was performed as described (Sowa et al., 2009), with modifications. Mouse neuro2a cells or rat DIV5 primary cortical neurons were infected with lentiviruses encoding individual FLAG-tagged baits. The neuronal synapsin1 promoter (Syn-pHAGE-N-FLAG vector) was used in cortical neurons. Puromycin resistance selected for stable expression in neuro2a. Lysates of neuro2a or DIV10 cortical neurons were subjected to immunoprecipitation using FLAG resin (Sigma), followed by 3XFLAG peptide elution (Sigma) and TCA precipitation. Proteins were trypsinized (Sequencing-Grade Trypsin, Promega) and washed (3M Empore C18 media), and tryptic peptides were loaded onto an LTQ linear ion trap mass spectrometer (ThermoFinnigan). Spectra were searched against target-decoy mouse or rat tryptic peptide databases. CompPASS analysis was done against dedicated neuro2a ( $>60$ bait-runs) or cortical neuron ( $>40$ bait-runs) IP-MS databases.

Plasmids. ORFs for IP-MS were subcloned by Gateway recombination into pHAGE-N-FLAG lentiviral vector. pRK7-FLAG-human TSC1 and TSC2 were from J. Blenis; pcdna3-FLAG-TSC2 Ser939Ala/Thr1462Ala was from Addgene. Rat Hap1 was amplified from P6 rat brain cDNA. shRNAs were expressed from pBluescript-U6 or subcloned into pLentiLox 3.7 for lentivirus. Rat shRNAs are as follows: Hap1i.1, GCTGCAGAGAGGAACGAAAGA; Hap1i.2, CACCGAAGATGATATCAAAGT; Hap1i.3, AGCTGAAACTGCTGGAAGAAG; Hap 1i.4, GCTCCTACATGCAGGATTATG; Tsc1i.1, CTAAAGAGCTTTCTGAGATCA. Mouse shRNAs are as follows: scrambled control, TACGCGCATAAGATTAGGGTA; Hapli.1, GAAGAAGATCACCGAAGATGA; Hapli.2, CACCGAAGATGATATCAAAGT; Tscli3, GATTATTACCTGGAAACCAAT.

Antibodies. Tsc1 (\#4906), Tsc2 (D93F12,\#4308), phosphoS6 235/236 (D57.2.2E, \#4858), total S6 (5G10,\#2217), and Erk (\#9102) antibodies were from Cell Signaling Technologies. Hap1 antibody (\#611302) was from BD Biosciences. Taul antibody (PC1C6, \#MAB3420) was from Millipore. The GFP antibody was produced in rabbit using recombinant GFP. The tubulin antibody (neuronal $\beta$-III, TUJ1, \#MMS-435P) was from Covance.

Coimmunoprecipitation analyses in HEK293T cells. Coimmunoprecipitations were performed as described (Litterman et al., 2011). Lysates were prepared in $1 \%$ Triton-X, $0.05 \%$ SDS, $0.025 \%$ deoxycholate, 150 mм NaCl, 50 mм Tris-HCl, 2 тм EDTA.

Hippocampal culture, transfection, lentiviral infection. Hippocampal cultures and transfections were done $2 \mathrm{~h}$ after plating as described (de la Torre-Ubieta et al., 2010). For biochemistry, neurons were plated at

$\leftarrow$

(Figure legend continued.) Tsc1. First and second panels from left, Lysates of HEK293T transfected with FLAG-TsC1 and Myc-Hap1B were subjected to immunoprecipitation followed by immunoblotting. Myc-Hap1 interacted with FLAG-Tsc1 and increased FLAG-Tsc1 protein abundance. Third panel from left, Lysates of HEK293T transfected with FLAG-Tsc2 and Myc-Hap1B were subjected to immunoprecipitation followed by immunoblotting. Right, Lysates of HEK293T transfected with FLAG-Tsc1, HA-Tsc2, and Myc-Hap1B were subjected to immunoprecipitation and immunoblotting. HA-Tsc2 was coexpressed to obtain equal levels of Tsc 1 because Hap1 expression increases Tsc1 protein abundance. C, Endogenous coimmunoprecipitation of Hap1 and Tsc1. Lysates of E18 mouse brain or neuro2a were subjected to immunoprecipitation followed by immunoblotting. D, Tsc1 deletion mutant coimmunoprecipitation analyses. Lysates of HEK293T transfected with FLAG-Tsc1 deletion mutants and Myc-Hap1B were subjected to immunoprecipitation using FLAG antibody, followed by immunoblotting using Myc antibody. Deletion of Tsc1 coiled-coil domain ( $\Delta C \mathrm{CC}$ ) but not Tsc2-binding domain ( $\Delta \mathrm{TSC}$ (2BD), Tsc1 $\mathrm{N}$ terminus ( $\Delta$ Nterm), or the C-terminal 167 amino acids (data not shown), reduced the interaction with Hap1, suggesting that interaction is mediated through Tsc1 coiled-coil domain. $\boldsymbol{E}$, Hap1 coimmunoprecipitation analyses. Lysates of HEK293T transfected with Myc-Hap1B coiled-coil (CC) fragments and FLAG-Tsc1 were subjected to immunoprecipitation using Myc antibody followed by immunoblotting using FLAG antibody. Hap1 coiled-coil domain 3 (CC3 alone) was sufficient to interact with Tsc1.
$1.0 \times 10^{6}$ cells/well, and lentiviral infection was performed $2 \mathrm{~h}$ after plating. Lysates were prepared in $1 \%$ Triton-X, 0.1\% SDS, 0.05 deoxycholate, $150 \mathrm{~mm} \mathrm{NaCl}, 50 \mathrm{~mm}$ Tris-HCl, 2 mм EDTA.

Hippocampal in utero electroporation. Electroporation of hippocampal germinal zone was performed as described (Navarro-Quiroga et al., 2007). E15 embryos of either sex were injected into the lateral ventricle (2 $\mu \mathrm{g} / \mu \mathrm{L}$ RNAi plasmid, $0.5 \mu \mathrm{g} / \mu \mathrm{L}$ pCAG-Venus-IRES-GFP) and electroporated along an ear-to-ear axis and slight rostrocaudal axis ( 6 pulses, 35 $\mathrm{V}$ for $50 \mathrm{~ms}, 950 \mathrm{~ms}$ intervals). Pups developed until P3. Brains were fixed in $4 \%$ sucrose/ $4 \%$ formaldehyde, and cryoprotected in 30\% sucrose; 40 $\mu \mathrm{m}$ coronal cryosections were prepared.

Immunostaining. Immunostaining was performed as described (de la Torre-Ubieta et al., 2010).

Statistical analyses. Statistics were performed in GraphPad Prism. Pairwise comparisons within multiple groups were done by two-way ANOVA followed by Bonferroni post hoc test. Percentage data in histograms are presented as mean \pm SEM.

\section{Results}

\section{Identification of Hap1 as a novel Tsc1 interactor}

To identify novel Tsc1 signaling mechanisms in neurons, we applied an IP-MS-based interaction proteomics approach in neural cells using CompPASS (Sowa et al., 2009). We expressed FLAGtagged Tsc1 in mouse neuro2a cells or primary rat cortical neurons. Lysates were subjected to immunoprecipitation using FLAG resin, and eluted trypsinized bait complexes were subjected to LC-MS/MS. The resulting protein assignments were analyzed in CompPASS, wherein we compared the set of proteins that copurified with Tsc1 against a large number of parallel IP-MS datasets generated in mouse neuro2a or primary rat cortical neurons. These analyses led to the identification of highconfidence interacting proteins distinct from background.

We found that Tsc1 copurified the proteins Tsc2 and Tbc1d7 as high-confidence interactors (Fig. $1 A$ ), which are known interactors of Tsc1 (Dibble et al., 2012), validating our IP-MS approach. We also uncovered the protein Hap1 as a novel highconfidence interactor of Tsc1 in both neuro2a cells and primary neurons (Fig. 1A). The expression of Hap1 is enriched in the brain and largely limited to nervous system cells (Dragatsis et al., 2000), suggesting that Hap1 may represent a neural cell-specific partner of Tscl.

We validated the Hap1-Tsc1 interaction. Exogenous Hap1 and Tscl coimmunoprecipitated in a reciprocal fashion in cells (Fig. 1B). Exogenous Hap1 also promoted the abundance of the coexpressed Tsc1 protein (Fig. 1B). Importantly, endogenous Hap1 and endogenous Tsc1 formed a complex in neuro2a cells and in mouse brain (Fig. 1C). Notably, a proportion, but not all, of endogenous Hap1 interacted with endogenous Tsc1. In other analyses, Hap 1 also copurified the Tsc1 partner protein Tsc2 (Fig. $1 B, C$ ), suggesting that Hap 1 forms a complex with the Tsc1-Tsc2 dimer.

We mapped the domains that mediate the interaction of Tsc1 and Hap1. Deletion of the entire $\mathrm{N}$-terminal region upstream of the Tsc1 coiled-coil domain (1-721), the Tsc2-binding region (302-430) (Hodges et al., 2001), or the C-terminal 167 amino acids (997-1164) of Tsc1 had little or no effect on the interaction with Hap1 (Fig. 1D; and data not shown). By contrast, deletion of the Tscl coiled-coil domain (721-997) reduced the interaction of Tsc1 with Hap1 (Fig. 1D). In analyses of Hap1, the Hap1 coiledcoil domain 3 (CC3; 354-417) was sufficient to interact with Tsc1. In contrast, coiled-coil domains 1 and 2 (CC1,2; 254-354) failed to associate with Tsc1 (Fig. 1E). Together, these data suggest that Hap 1 and Tsc1 interact in cells and in the brain, and that the interaction occurs through coiled-coiled domains. 
A

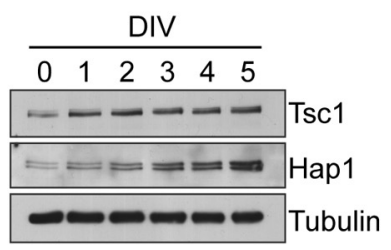

C

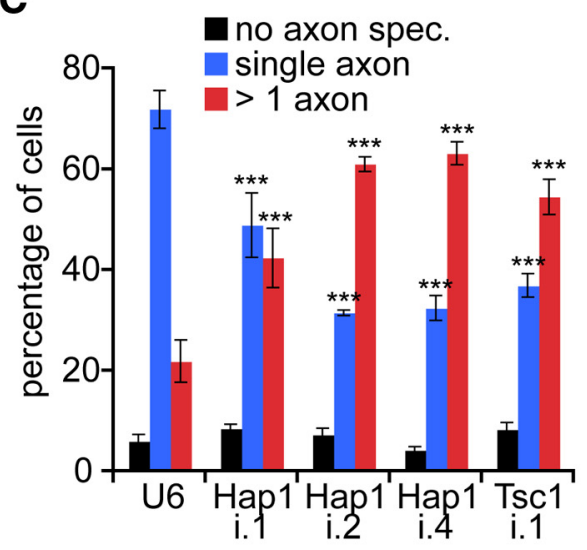

E
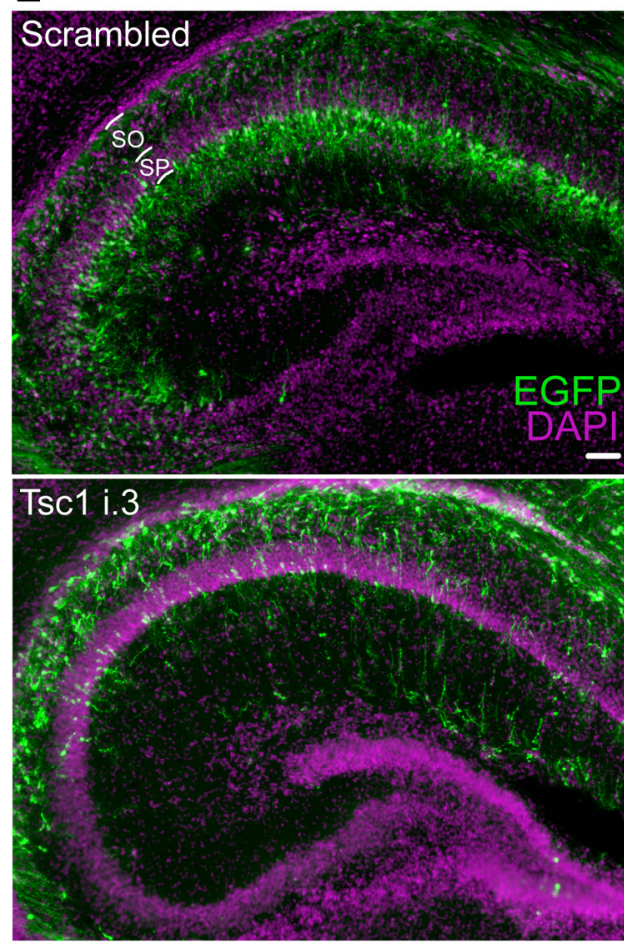

B
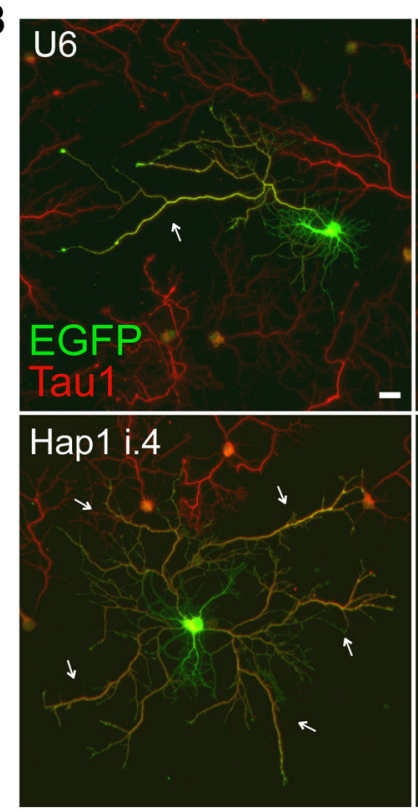

.

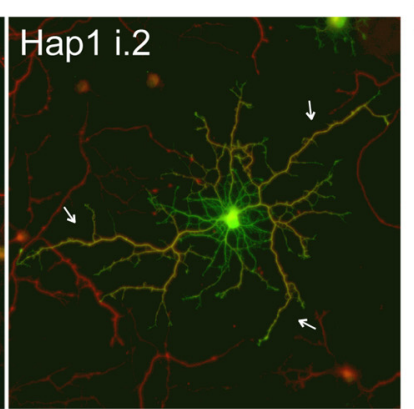

D
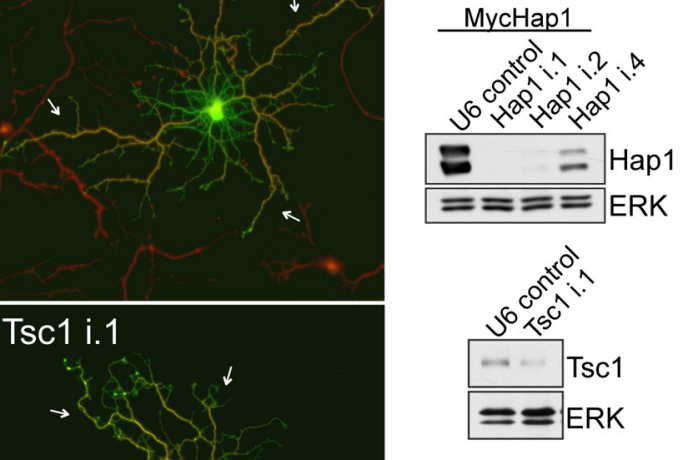

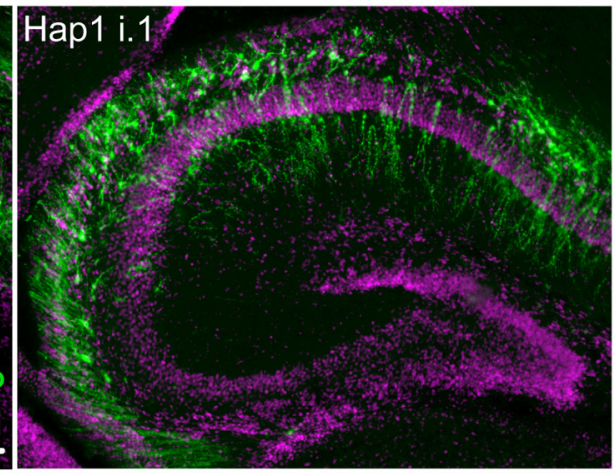

$\mathbf{F}$

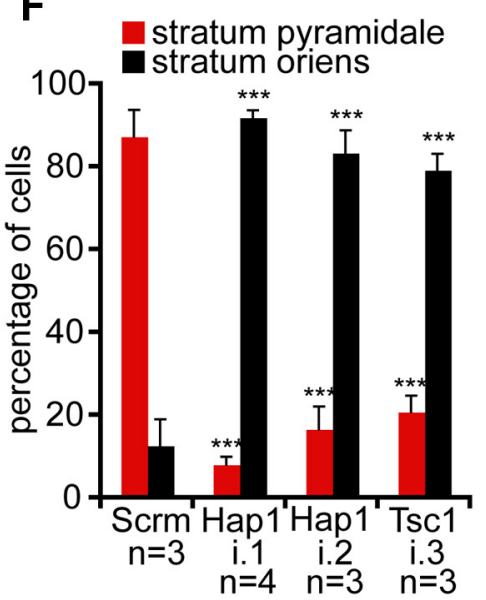

G

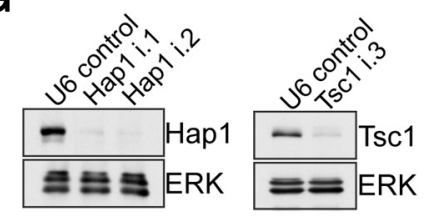

Figure 2. Hap1 and Tsc1 regulate hippocampal pyramidal neuron axon specification and positioning. $A$, Lysates of hippocampal neurons from 0 to 5 DIV were subjected to immunoblotting using the Tsc1, Hap1, or Tubulin ( $\beta$-III type) antibody. B, E18 rat hippocampal neurons transfected $2 \mathrm{~h}$ after plating with the control U6 RNAi or Hap1 RNAi or Tsc1 RNAi plasmids were subjected to immunocytochemistry on DIV5 using the GFP (green) and the axonal antigen Tau1 (red) antibodies. Scale bar, $20 \mu \mathrm{m}$. The i.1, i.2, i.4 nomenclature refers to individual shRNAs. C, Quantification of axon specification as percentage of neurons. Hap1 or Tsc 1 knockdown promoted specification of supernumerary axons ( $n=3$ experiments). ${ }^{* * *} p<0.001$ (two-way ANOVA and post hoc Bonferroni test). No axon spec., neurons that failed to specify a Tau1-immunoreactive axon. D, Lysates of HEK293T cells transfected with Myc-Hap1 and control U6 RNAi or Hap1 RNAi (top) or DIV5 hippocampal neurons infected $2 \mathrm{~h}$ after plating with U6 RNAi or Tsc1 RNAi (bottom) were subjected to immunoblotting. $E$, E15 mice were electroporated with Hap1 RNAi, Tsc1 RNAi, or scrambled control RNAi and VENUS-IRES-EGFP plasmids. Brains were harvested on P3 and subjected to immunohistochemistry using the GFP (green) antibody and Hoechst 33258 (purple). Scale bar, $50 \mu \mathrm{m}$. SP, Stratum pyramidale; $S 0$, stratum oriens. $F$, Quantification of neuronal position as percentage of neurons. Knockdown of Tsc1 or Hap1 impaired neuronal positioning into the SP in the hippocampus in vivo $(n \geq 3) .{ }^{* * *} p<0.001$ (two-way ANOVA and posthoc Bonferroni test). Number of animals analyzed per condition is shown. G, Lysates of mouse neuro2a cells transfected for $3 \mathrm{~d}$ with control U6 RNAi, Hap1 RNAi, or Tsc1 RNAi were subjected to immunoblotting.

Hap1 regulates axon specification and positioning of hippocampal pyramidal neurons

Having identified Hap 1 as a Tsc1 interactor, we asked whether these proteins shared biological roles in neurons. Both Tsc1 and Hap1 were expressed in hippocampal neurons (Fig. 2A). Their levels increased from DIV0 to DIV5 (Fig. 2A), which coincides temporally with the specification and outgrowth of axons. To probe for a role of Hap1 in axon development, we 
A

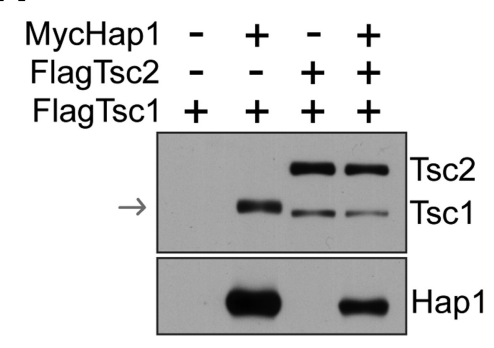

B
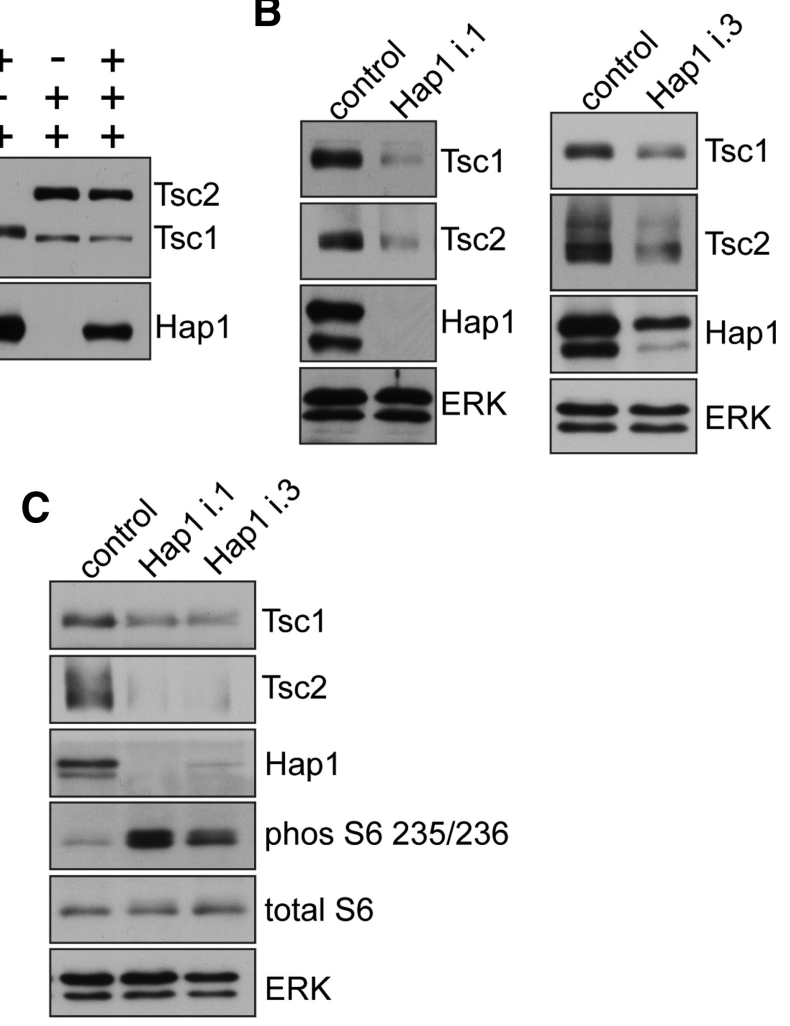

Figure 3. Hap1 controls Tsc1 abundance and S6 phosphorylation. A, Lysates of HEK293T cells transfected with FLAG-Tsc1 alone or FLAG-Tsc1 together with FLAG-Tsc2 or Myc-Hap1B were subjected to immunoblotting. Coexpression of Myc-Hap1B or FLAGTsc2 promoted FLAG-Tsc1 abundance. Arrow indicates location of Tsc1. B, Lysates of DIV5 hippocampal neurons infected on DIV0 $2 \mathrm{~h}$ after plating with Hap1 RNAi or control lentivirus cultured under basal conditions were subjected to immunoblotting. Hap1 knockdown triggered downregulation of Tsc1 and Tsc2. The i.1, i.3 nomenclature refers to individual shRNAs. C, Lysates of DIV5 hippocampal neurons infected on DIVO $2 \mathrm{~h}$ after plating with RNAi cultured under basal conditions were subjected to immunoblotting. Hap 1 knockdown triggered downregulation of Ts 1 and $\mathrm{TsC} 2$ and stimulated phosphorylation of the mTORC1 effector 56 .

used a plasmid-based method of RNAi (Gaudilliere et al., 2002). Knockdown of Hap1 in hippocampal neurons promoted the specification of more than one axon per neuron (Fig. $2 B, C$ ). The Hap 1 knockdown-induced phenotype recapitulated the effect of Tsc1 knockdown, which led to the specification of supernumerary axons (Fig. $2 B, C$ ) (Choi et al., 2008). These data suggest that Hap 1 and Tscl suppress axon specification in hippocampal neurons.

We determined the role of Hap1 in hippocampal neuron development in vivo. Because the establishment of axon-dendrite polarity is critical for proper migration and positioning of developing pyramidal neurons (Barnes and Polleux, 2009; de la TorreUbieta and Bonni, 2011), we reasoned that Hap1 and Tsc1 might influence neuronal positioning in the hippocampus in vivo. Using in utero electroporation in embryonic day 15 (E15) mice, we found that pyramidal neurons in control mice at postnatal day 3 (P3) completed migration through the stratum oriens and incorporated into the pyramidal cell layer (Fig. $2 E, F)$. In contrast, pyramidal neurons in Hap 1 knockdown animals failed to reach the pyramidal cell layer, and their soma remained within the stratum oriens (Fig. 2 E, F). Notably, Tsc1 knockdown phenocopied the impairment of pyramidal neuron positioning in the hippocampus induced by Hap 1 knockdown (Fig. 2E,F). Collectively, our data suggest that both Hap1 and Tsc1 regulate axon-dendrite morphogenesis and positioning of hippocampal pyramidal neurons.

\section{Hap1 regulates Tsc1 abundance and mTORC1 signaling in neurons}

We next determined a mechanism by which Hap1 might regulate Tsc1 function in cells. Tsc1 protein is not expressed at a high level in cells in the absence of coexpressed Tsc2 resulting from low solubility and stability (Nellist et al., 1999). Expression of Hap1 strongly promoted the abundance of coexpressed Tsc1, mimicking the effect of Tsc2 coexpression (Figs. $1 B$ and $3 A$ ). Importantly, lentivirus-mediated knockdown of Hap1 triggered downregulation of endogenous Tsc1 in hippocampal neurons (Fig. 3B). The extent of Tsc1 downregulation correlated with the extent of Hap1 knockdown (Fig. 3B). Because Tsc1-Tsc2 is an essential negative regulator of $\mathrm{mTORC1}$ signaling, we assessed the role of Hap1 in regulating the $\mathrm{mTORC1}$ pathway in neurons. Knockdown of Hapl in hippocampal neurons stimulated phosphorylation of the mTORC1 effector protein S6 (Fig. 3C). Together, these data suggest that Hap1 promotes the abundance of Tsc1 and inhibits neuronal mTORC1 signaling.

We next assessed the role of mTORC1 signaling on Hap1-mediated control of hippocampal neuron development. Inhibition of mTORC1 by rapamycin suppressed the specification of supernumerary axons induced by Hap1 knockdown and restored single-axon polarity in these neurons (Fig. $4 A, B$ ). Rapamycin had little or no effect on single axon specification in control neurons (Fig. 4B), suggesting a specific suppression of the Hapl knockdown effect. In complementary experiments, inhibition of mTORC1 by expression of an active form of Tsc2, in which inhibitory Akt phosphorylation sites (Ser939/Thr1462) were replaced with alanine (Tsc2 ${ }^{\text {SA/TA }}$ ) (Manning et al., 2002), suppressed the specification of supernumerary axons and restored single-axon polarity in Hap1 knockdown neurons (Fig. 4C,D). Together, these data suggest that Hap1 regulates neuronal morphogenesis upstream of neuronal mTORC1, by promoting Tsc1 function and inhibiting mTORC1 signaling.

\section{Discussion}

In this study, we have identified a novel Hap1-Tsc1 signaling link that controls the mTORC1 pathway in neurons. Accordingly, depletion of Hap1 promotes specification of supernumerary axons in hippocampal neurons and profoundly impairing the positioning of hippocampal pyramidal neurons in vivo. We have also elucidated a mechanism by which Hap 1 regulates neuronal Tsc1 function. Hap1 knockdown triggers the downregulation of Tscl and activates the $\mathrm{mTORC1}$ signaling pathway in hippocampal neurons. Consistent with these findings, inhibition of mTORC1 signaling suppresses the Hap1 knockdowninduced axon phenotype in neurons. Our findings uncover Hap1 as a novel regulator of neuronal Tsc1-Tsc2/mTORC1 signaling and define a critical role for the Hap1-Tsc1 interaction in the control of axon development and neuronal positioning in the brain. 
Hap1 represents, to our knowledge, the first identified neural-specific molecular regulator of $\mathrm{mTORC1}$ signaling. This finding suggests the interesting possibility that disruption of neuron-specific regulators of mTORC1 signaling might contribute to the pathogenesis of neurological manifestations of TSC, including autism, intellectual disability, and epilepsy.

The identification of Hap1 as a novel interactor of Tsc1 should shed light on the regulation of the neuronal Tsc1-Tsc2 complex. Tsc1 and Tsc2 depend on each other for their solubility and stability, and loss of either protein or dissociation of the Tsc1-Tsc2 dimer impairs mTORC1 signaling (Orlova and Crino, 2010; Dibble et al., 2012). Studies in non-neural cells suggest that the Tsc1-Tsc2 complex also contains the protein Tbcld7 (Nakashima et al., 2007; Dibble et al., 2012). Our IP-MS analyses revealed that Tbcld7 also interacts with Tscl in neurons, suggesting that the Tsc1-Tbc1d7-Tsc2 complex may form in neurons. It will be interesting to determine whether Hap1 might cooperate with Tbcld7 or Tsc 2 to regulate the abundance of Tscl in neurons and thereby regulate neuronal mTORC1 signaling.

Tsc1-Tsc2 and mTORC1 pathway components control axon development and pyramidal neuron morphogenesis (Han and Sahin, 2011). Loss of Tsc1-Tsc2 leads to abnormal axon development and neuronal polarization and positioning, along with dendritic arbor defects in the cerebral cortex (Choi et al., 2008; Li et al., 2008; Feliciano et al., 2011; Goto et al., 2011). Our study suggests Hapl regulates pyramidal neuron axon specification and positioning and strengthens the link between axon-dendrite polarization and neuron positioning.

The identification of an intimate Hap1Tscl connection bears implications for both Hap1 and mTORC1 signaling beyond the control of axon morphogenesis and neuronal positioning. It will be interesting to determine the role of Hap1 in the control of long-term depression and hippocampal excitatory/ inhibitory balance, which are regulated by Tsc1 (Bateup et al., 2013). Conversely, it will be interesting to investigate whether Tsc1 may influence Hap1 functions, including regulation of $\mathrm{GABA}_{\mathrm{A}}$ receptor surface expression (Rong et al., 2007).

Our study demonstrates that multibait IP-MS interaction proteomics approaches can be performed in primary neurons. Prior similar efforts on Tsc1 and other proteins implicated in autism have used non-neural cells (Guo et al., 2010; Sakai et al., 2011). Using CompPASS, we have identified high-confidence interactions in endogenous neural proteomes. It will be interesting to apply similar IP-MS approaches to human neuronal proteomes, including in patient-derived neurons.

Loss of TSC1 protein function in the brain in TSC manifests as autism, epilepsy, and ID. Our study reveals that Hap1 controls Tsc1 function in neurons, raising the possibility that Hap 1 may play a role in the control of cognitive and neurological function. Remarkably, recent whole-exome sequencing has identified a rare complete Hap1 knock-out among patients with ASD (Lim et al., 2013), suggesting that Hap1 may be relevant to ASD pathogenesis. Elucidation of a Hap1-TSC1 signaling link in ASD may expand our understanding of the molecular components at play in ASD pathogenesis.

\section{References}

Barnes AP, Polleux F (2009) Establishment of axon-dendrite polarity in developing neurons. Annu Rev Neurosci 32:347-381. CrossRef Medline

Bateup HS, Johnson CA, Denefrio CL, Saulnier JL, Kornacker K, Sabatini BL (2013) Excitatory/inhibitory synaptic imbalance leads to hippocampal hyperexcitability in mouse models of tuberous sclerosis. Neuron 78:510 522. CrossRef Medline

Choi YJ, Di Nardo A, Kramvis I, Meikle L, Kwiatkowski DJ, Sahin M, He X (2008) Tuberous sclerosis complex proteins control axon formation. Genes Dev 22:2485-2495. CrossRef Medline

de la Torre-Ubieta L, Bonni A (2011) Transcriptional regulation of neuro- 
nal polarity and morphogenesis in the mammalian brain. Neuron 72:2240. CrossRef Medline

de la Torre-Ubieta L, Gaudillière B, Yang Y, Ikeuchi Y, Yamada T, DiBacco S, Stegmüller J, Schüller U, Salih DA, Rowitch D, Brunet A, Bonni A (2010) A FOXO-Pak1 transcriptional pathway controls neuronal polarity. Genes Dev 24:799-813. CrossRef Medline

Dibble CC, Elis W, Menon S, Qin W, Klekota J, Asara JM, Finan PM, Kwiatkowski DJ, Murphy LO, Manning BD (2012) TBC1D7 is a third subunit of the TSC1-TSC2 complex upstream of mTORC1. Mol Cell 47:535-546. CrossRef Medline

Dragatsis I, Dietrich P, Zeitlin S (2000) Expression of the Huntingtinassociated protein 1 gene in the developing and adult mouse. Neurosci Lett 282:37-40. CrossRef Medline

Feliciano DM, Su T, Lopez J, Platel JC, Bordey A (2011) Single-cell Tsc1 knockout during corticogenesis generates tuber-like lesions and reduces seizure threshold in mice. J Clin Invest 121:1596-1607. CrossRef Medline

Gaudilliere B, Shi Y, Bonni A (2002) RNA interference reveals a requirement for myocyte enhancer factor $2 \mathrm{~A}$ in activity-dependent neuronal survival. J Biol Chem 277:46442-46446. CrossRef Medline

Goto J, Talos DM, Klein P, Qin W, Chekaluk YI, Anderl S, Malinowska IA, Di Nardo A, Bronson RT, Chan JA, Vinters HV, Kernie SG, Jensen FE, Sahin M, Kwiatkowski DJ (2011) Regulable neural progenitor-specific Tsc1 loss yields giant cells with organellar dysfunction in a model of tuberous sclerosis complex. Proc Natl Acad Sci U S A 108:E1070-E1079. CrossRef Medline

Guo L, Ying W, Zhang J, Yuan Y, Qian X, Wang J, Yang X, He F (2010) Tandem affinity purification and identification of the human TSC1 protein complex. Acta Biochim Biophys Sin (Shanghai) 42:266-273. CrossRef Medline

Han JM, Sahin M (2011) TSC1/TSC2 signaling in the CNS. FEBS Lett 585: 973-980. CrossRef Medline

Hodges AK, Li S, Maynard J, Parry L, Braverman R, Cheadle JP, DeClue JE, Sampson JR (2001) Pathological mutations in TSC1 and TSC2 disrupt the interaction between hamartin and tuberin. Hum Mol Genet 10:28992905. CrossRef Medline

Li XJ, Li SH, Sharp AH, Nucifora FC Jr, Schilling G, Lanahan A, Worley P, Snyder SH, Ross CA (1995) A huntingtin-associated protein enriched in brain with implications for pathology. Nature 378:398-402. CrossRef Medline

Li YH, Werner H, Püschel AW (2008) Rheb and mTOR regulate neuronal polarity through Rap1B. J Biol Chem 283:33784-33792. CrossRef Medline

Lim ET, Raychaudhuri S, Sanders SJ, Stevens C, Sabo A, MacArthur DG, Neale BM, Kirby A, Ruderfer DM, Fromer M, Lek M, Liu L, Flannick J,
Ripke S, Nagaswamy U, Muzny D, Reid JG, Hawes A, Newsham I, Wu Y, et al. (2013) Rare complete knockouts in humans: population distribution and significant role in autism spectrum disorders. Neuron 77:235242. CrossRef Medline

Litterman N, Ikeuchi Y, Gallardo G, O'Connell BC, Sowa ME, Gygi SP, Harper JW, Bonni A (2011) An OBSL1-Cul7Fbxw8 ubiquitin ligase signaling mechanism regulates Golgi morphology and dendrite patterning. PLoS Biol 9:e1001060. CrossRef Medline

Ma XM, Blenis J (2009) Molecular mechanisms of mTOR-mediated translational control. Nat Rev Mol Cell Biol 10:307-318. CrossRef Medline

Manning BD, Tee AR, Logsdon MN, Blenis J, Cantley LC (2002) Identification of the tuberous sclerosis complex-2 tumor suppressor gene product tuberin as a target of the phosphoinositide 3-kinase/akt pathway. Mol Cell 10:151-162. CrossRef Medline

Nakashima A, Yoshino K, Miyamoto T, Eguchi S, Oshiro N, Kikkawa U, Yonezawa K (2007) Identification of TBC7 having TBC domain as a novel binding protein to TSC1-TSC2 complex. Biochem Biophys Res Commun 361:218-223. CrossRef Medline

Navarro-Quiroga I, Chittajallu R, Gallo V, Haydar TF (2007) Long-term, selective gene expression in developing and adult hippocampal pyramidal neurons using focal in utero electroporation. J Neurosci 27:5007-5011. CrossRef Medline

Nellist M, van Slegtenhorst MA, Goedbloed M, van den Ouweland AM, Halley DJ, van der Sluijs P (1999) Characterization of the cytosolic tuberinhamartin complex: tuberin is a cytosolic chaperone for hamartin. J Biol Chem 274:35647-35652. CrossRef Medline

Orlova KA, Crino PB (2010) The tuberous sclerosis complex. Ann N Y Acad Sci 1184:87-105. CrossRef Medline

Rong J, Li SH, Li XJ (2007) Regulation of intracellular HAP1 trafficking. J Neurosci Res 85:3025-3029. CrossRef Medline

Sakai Y, Shaw CA, Dawson BC, Dugas DV, Al-Mohtaseb Z, Hill DE, Zoghbi HY (2011) Protein interactome reveals converging molecular pathways among autism disorders. Sci Transl Med 3:86ra49. CrossRef Medline

Santini E, Huynh TN, MacAskill AF, Carter AG, Pierre P, Ruggero D, Kaphzan H, Klann E (2013) Exaggerated translation causes synaptic and behavioural aberrations associated with autism. Nature 493:411-415. CrossRef Medline

Sheng G, Xu X, Lin YF, Wang CE, Rong J, Cheng D, Peng J, Jiang X, Li SH, Li XJ (2008) Huntingtin-associated protein 1 interacts with Ahil to regulate cerebellar and brainstem development in mice. J Clin Invest 118: 2785-2795. CrossRef Medline

Sowa ME, Bennett EJ, Gygi SP, Harper JW (2009) Defining the human deubiquitinating enzyme interaction landscape. Cell 138:389-403. CrossRef Medline 